\title{
Bubble Performance Study before and after Detachment in a Membrane Bioreactor
}

\author{
Guiqin Liu, Hui An, Peng Cheng Wang, Joo Guan Hang, Simon C.M. Yu
}

\begin{abstract}
In the membrane filtration system of the Membrane Bioreactor, air bubbles are generated in the Membrane Bioreactor tank to scour the surface of the hollow fiber membranes by creating shear forces and vibrations to prevent fouling, known as air scouring process. The generation and formation characteristics of the air bubbles have a significant impact on the performance of the overall MBR system. In this paper, experimental studies were performed to investigate the bubble formation characteristics before and after detachment. Before detachment, based on the correlation between horizontal diameter and vertical diameter, two stages are identified, namely bound expansion stage and acceleration expansion. In the bound expansion stage, the bubble rises at a constant speed, while expands uniformly. In the acceleration expansion stage, the bubbles still rising at a constant speed, while expands speed gradually slows down. The higher the air inlet velocity, the shorter the bound expansion stage. After detachment, the bubble rising behavior is observed to be classified into three regions. In the first and third region, the bubble rising speed is constant, while in the second region, the bubble rising unsteadily. At detachment time, the bubble size in the horizontal direction does not change with air inlet speed, while increase slightly in the vertical direction.
\end{abstract}

Keywords - Two-Phase Flow, Bubble generation, Experimental study, Membrane Bioreactor (MBR).

\section{INTRODUCTION}

A IR can be injected into the Membrane bioreactor (MBR) in the format of bubbles, in order to control fouling of the membrane, known as air scouring process. During this process, the compressed air flows into the aeration diffuser branch pipes and exits through the aeration diffuser nozzles on top of the branch pipes, forming air bubbles. The air bubbles generated will rise upwards, flowing past and through the membrane networks and scouring the membrane surfaces. The shape and speed of the bubbles generated directly affect the surface shear on the membrane surface which then determines the cleaning efficiency of the overall aeration diffuser system.

The air bubble shape varies significantly in different flow regimes depending on some non-dimensional parameters such as Reynolds number, Eötvös number and Morton number [1]. Reynolds number is a measure of the flow pattern, the Eötvös number is a measure of the volume of the bubble, while the Morton number represents the physical properties of the fluid. In general, air bubbles can be categorized into three different shapes, namely Spherical, Ellipsoidal and Spherical Cap based on Reynolds number and Eötvös number. Fig. 1 shows how the

Guiqin Liu is with the Singapore Institute of Technology, Singapore (phone: 65-65921063; e-mail: guiqin.liu@ singaporetech.edu.sg).

Hui An is with the Singapore Institute of Technology, Singapore (phone: 65 65922074; e-mail: hui.an@ singaporetech.edu.sg).

Peng Cheng Wang is with the Singapore Institute of Technology, Singapore (phone: 65-65928510; e-mail: victor.wang@singaporetech.edu.sg). values of these dimensionless numbers affect the shape of the bubble. Studies from Bhaga and Weber [2] showed that small bubbles, which have low Reynolds or Eötvös number $(\operatorname{Re} \approx 1$ or Eo $<1$ ), rise in a steady fashion and maintain the spherical shape. The shape of larger bubbles, with intermediate Reynolds number and Eötvös number $(1<\operatorname{Re}<100$ and $1<$ Eo < 100), are significantly affected by the flow conditions. They tend to form ellipsoidal bubbles. The bubbles will still rise steadily in the liquid along a straight path despite the different bubble shapes. With further increase in Reynolds number and Eötvös number, the air bubbles will form the shape of spherical cap [3].

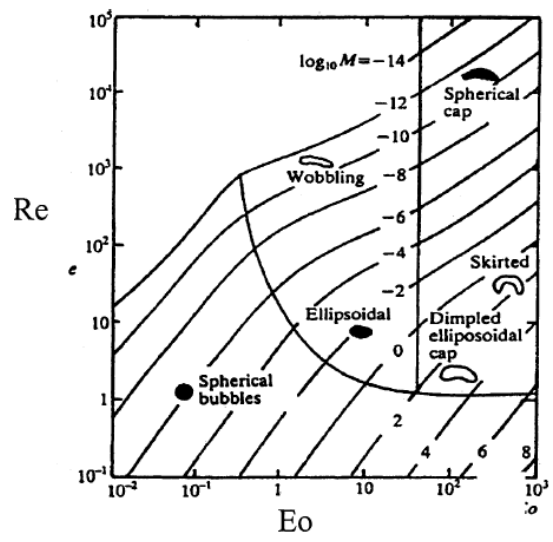

Fig. 1: Regime map of rising bubble shape in liquids

The bubble size at the point of detachment from the nozzle depends on several factors, including nozzle diameter and air flow rate. The influence of nozzle diameter on the bubble size is negligible at relatively high flow rates and becomes significant at low flow rates [4]. The influence of the air flow rate is insignificant at low flowrate, while proportional to the cubic root of gas flow rate at high flow rate. However, when the gas flow rate exceeds certain values, sludge flow happens and it is impossible to obtain air bubbles.

The terminal bubble rising speed is dominated by the force acting on it. At steady state (rising speed does not change with time), the speed can be calculated based on the below equation: $u_{\infty}^{2}=\frac{4\left(\rho_{l}-\rho_{g}\right) g D_{b}}{3 C_{D} \rho_{l}}$

where $D_{b}$ is the bubble diameter, $\rho_{l}$ and $\rho_{g}$ are liquid and gas densities, $u_{\infty}$ is the bubble terminal velocity, and $C_{D}$ is the

Joo Guan Hang is with the Singapore Institute of Technology, Singapore (phone: 65-65922144; e-mail: jooguan.hang@ singaporetech.edu.sg).

Simon C.M. Yu was with the Nanyang Technological University, Singapore and Singapore Institute of Technology, Singapore. Now he is with the Hong Kong Polytechnic University, Hong Kong ( e-mail: simon.c.yu@polyu.edu.hk) 
bubble drag coefficient. For water, $C_{D}$ is 2.65 if the bubble size is $8-50 \mathrm{~mm}$ [5].

Bubble formation stage is important to the bubble performance, such as the shape, size and speed of the air bubbles. Under constant gas flow rate, the bubble formation stage includes bound expansion and acceleration expansion [6]. In the bound expansion stage, the bubble grows radially due to the incoming gas flow, but the bubble base remains attached to the orifice. During the acceleration expansion stage, the bubble lifts away from the orifice but is still attached to it through a thin string, called a neck. At the end of the second stage, the neck pinches off and the bubble detaches at the time when the neck length is equal to or larger than the orifice diameter. Other researchers showed the detachment happens when the neck length is $40 \%$ of the instantaneous bubble diameter [4].

The factors affecting the bubble formation was summarized by Amol and Jyeshtharaj [7], including gas flow rate, orifice size and liquid properties (viscosity, density etc). The property of the gas on the bubble generation was studied and results showed that the bubble size depends on the surface tension and gas density, while the bubble frequency depends on the contact angle and the surface tension [8]. The bubble size increase with the gas velocity in all the $\mathrm{x}, \mathrm{y}$ and $\mathrm{z}$ direction [9].

In this paper, experimental study was carried out to study the bubble formation characteristics, in particular the air bubble size and rising speed.

\section{II.EXPERIMENTAL SETUP}

The experimental apparatus is equipped with a square aeration diffuser pipe placed horizontally in the aeration diffuser water tank. The side length of the aeration pipe is 60 $\mathrm{mm}$ with an overall length $500 \mathrm{~mm}$. The pipe has seven orifices on the top surface with different diameters of $2, \mathrm{~mm}, 3 \mathrm{~mm}, 4$ $\mathrm{mm}, 5 \mathrm{~mm}, 6 \mathrm{~mm}, 8 \mathrm{~mm}$ and $10 \mathrm{~mm}$ respectively. The center to center distance between two adjacent orifices is kept at 50 $\mathrm{mm}$. The water tank is $500 \mathrm{~mm}$ long, $300 \mathrm{~mm}$ wide and $500 \mathrm{~mm}$ high. The aeration pipe is centralized at $150 \mathrm{~mm}$ above the bottom of the water tank. Both the aeration diffuser pipe and the water tank are made of transparent acrylic, in order to capture the bubble performance characteristics with the use of a high speed camera.

The elevation of the free surface is controlled by the water dosing system from the bottom of the tank. Water is dosed into the water tank automatically when the elevation is below the settings. The accuracy of the Water level sensor is $\pm 1 \mathrm{~mm}$.

The compressed air is supplied from the centralized gas pipe system through a pressure regulator set at $2.7 \mathrm{bar}$, which is the average pressure where bubbles are released in the MBR. The flow rate is regulated with the use of five flowmeters (range of 0-1 SLM, 0-10 SLM, 0-50 SLM and two 0-100 SLM), in order to control the flow rate in both small and large range with acceptable accuracy.

High speed camera (Photron MINI UX100) is used to capture the air bubble formations. Photron FASTCAM Analysis software is used to capture the bubble size and location.

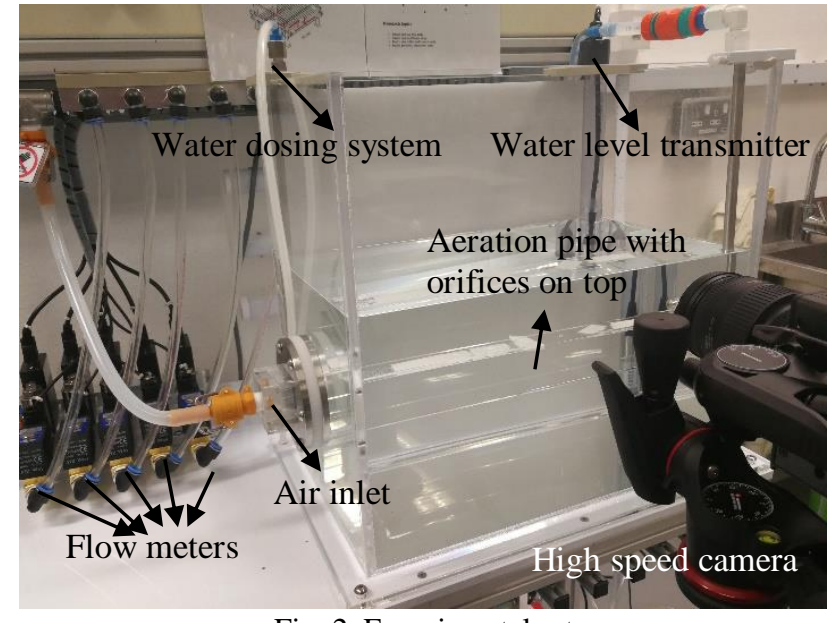

Fig. 2. Experimental setup

\section{EXPERIMENTAL METHODS}

During the experiment, all the orifices except the target diameter orifice are closed by tape. After the air flow rate is regularted and stadalized, bubbles from the target orifice are captured with the high speed camera at a frame rate of $4000 \mathrm{fps}$ and resolution of $312 \times 1280$ pixes. Bubbles generated are caputured for 7 seconds, with at least 50 bubbles, depending on the air flow rate/speed.

Bubble size is measured at the detaching time in both vertical and horizontal directions. Averaged value of 20 bubbles will be reported.

In order to measure the bubble's rising speed before or after detachment, a target point is set first. For the speed before detachment, the most top point of the bubble was used, while for the speed after detachment, the most right point along the maximum diameter line is selected as the capturing point, and its vertical location is recorded to calculate the rising speed. However, one challenge that was encountered during the capturing process is the target point, which could not accurately captured due toe picture contrast and turbulence of the bubble. As a result, the instantaneous speed may not be representable. Hence, in this section, the location of the air bubble is recorded and the speed was calculated based on the slope of the location vs time curve.

\section{RESULTS AND DISCUSSION}

\section{A. Bubble performance before detaching}

As the capturing point is set on the top of the bubble as shown in Fig. 3, the speed at the centre of the bubbles is estimated to be half of the captured speed. The measurement is taken from the time when the top of the bubble is released from the nozzle to the time when the bubble is fully detached, including both the bound stage and acceleration stage.

The vertical location measured on the top of several bubbles before detachment are illustrated in Fig. 4 at an air inlet velocity of $1 \mathrm{~m} / \mathrm{s}$. During this period, the bubble grows at almost a constant speed. The averaged rising speed on the top point of the bubbles is calculated to be $0.42 \mathrm{~m} / \mathrm{s}$, indicating an average speed at the centre of the bubbles $V_{\text {bubble }}$ of $0.21 \mathrm{~m} / \mathrm{s}$. For the 
case of air inlet velocity of $2 \mathrm{~m} / \mathrm{s}$, the $V_{\text {bubble }}$ is $0.22 \mathrm{~m} / \mathrm{s}$ as depicted in Fig. 5. It can be conclued that the bubble rising speed does not chanage significantly with the air inlet velocity.

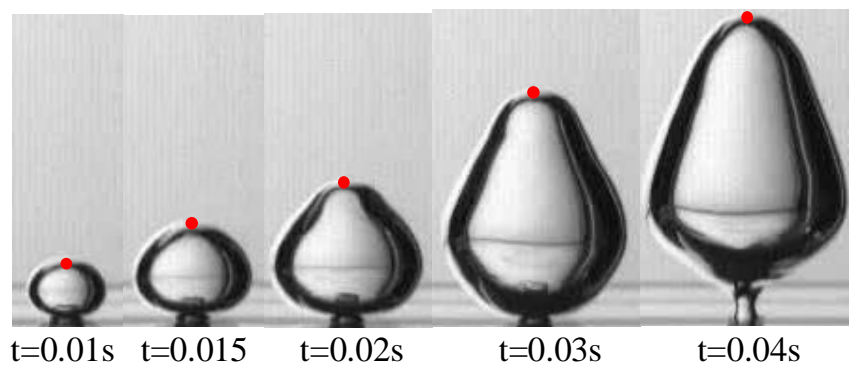

Fig. 3. Development of bubble shape before detaching at $1 \mathrm{~m} / \mathrm{s}$

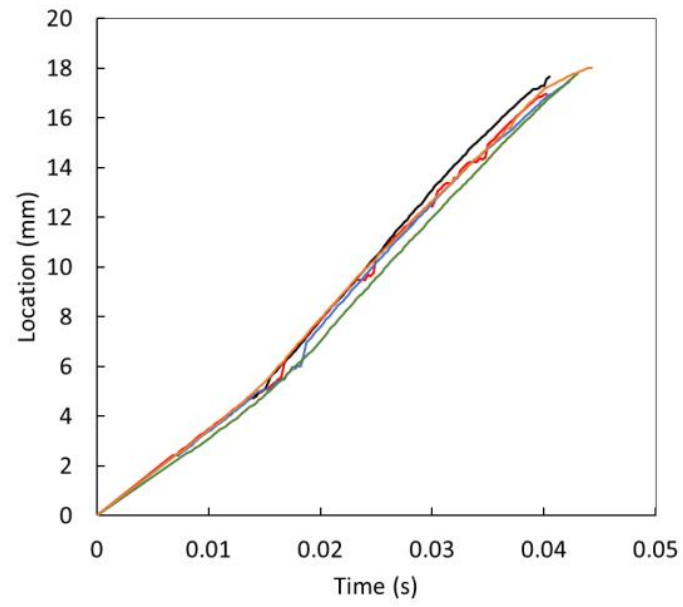

-bubble 1 -bubble 2 -bubble 3 -bubble 4 - bubble 5

Fig. 4. Bubble rising speed before detaching for $2 \mathrm{~mm}$ orifice at $1 \mathrm{~m} / \mathrm{s}$

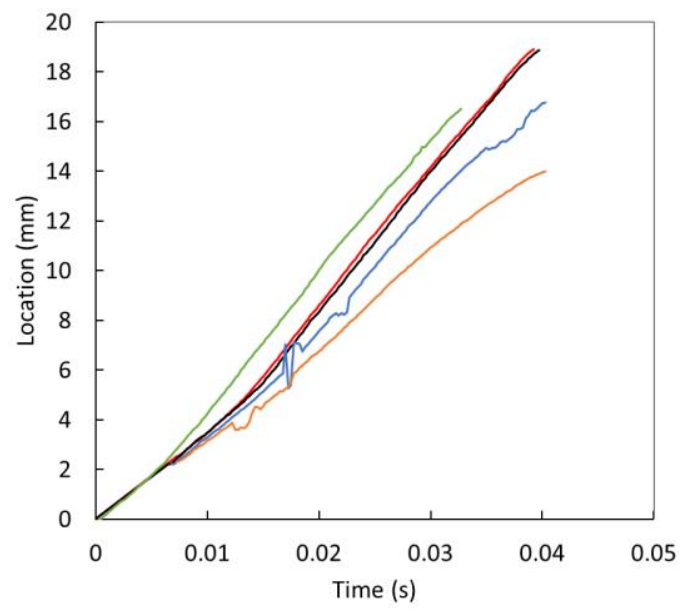

-bubble 1 -bubble 2 - bubble 3 - bubble 4 - bubble 5

Fig. 5. Bubble rising speed before detaching for $2 \mathrm{~mm}$ orifice at $2 \mathrm{~m} / \mathrm{s}$

The relationship between the horizontal diameter and vertical height of the bubble top point is shown in Fig. 6 and Fig. 7. The two stages are clearly indicated. In the Bound expansion stage, the bubble speed is equal to the bubble radial expansion speed, which agrees well with the previous research results by Zhang
[6]. The near spherical shape is also observed from Fig. 3 at time intervals of $0.01 \mathrm{~s}, 0.015 \mathrm{~s}$ and $0.02 \mathrm{~s}$. In the acceleration expansion stage, the bubble is still attached to the nozzle orifice through a neck, but either the neck or the bubble will be elongated as shown in Fig. 3 at time of $0.03 \mathrm{~s}$ and $0.04 \mathrm{~s}$. The criteria time of the two stages is $0.2 \mathrm{~s}$ for the $1 \mathrm{~m} / \mathrm{s}$ air inlet case, while $0.15 \mathrm{~s}$ for the $2 \mathrm{~m} / \mathrm{s}$ air ininlet case, indicating that the bubble bound expansion stage is shorter with higher air inlet velocity.

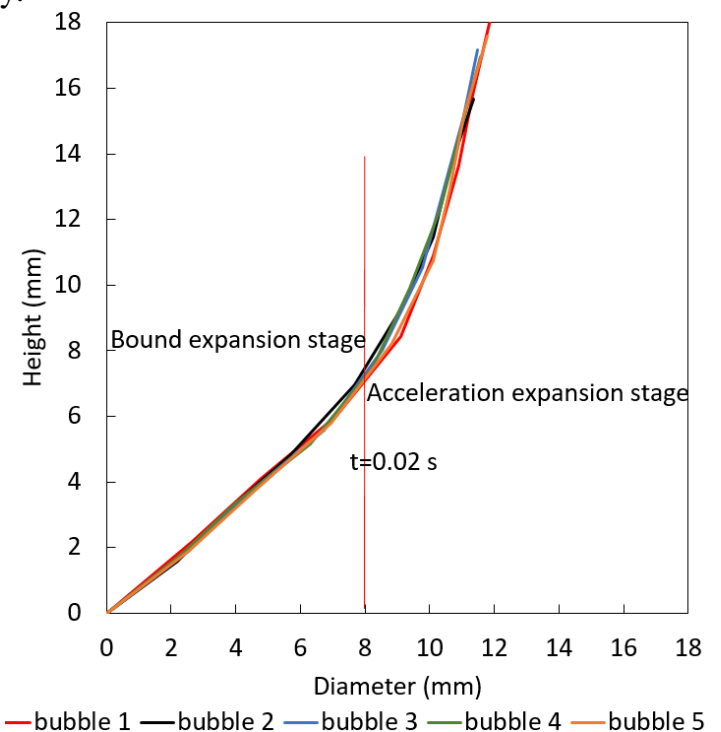

Fig. 6. Correlation between bubble diameter and height at $1 \mathrm{~m} / \mathrm{s}$

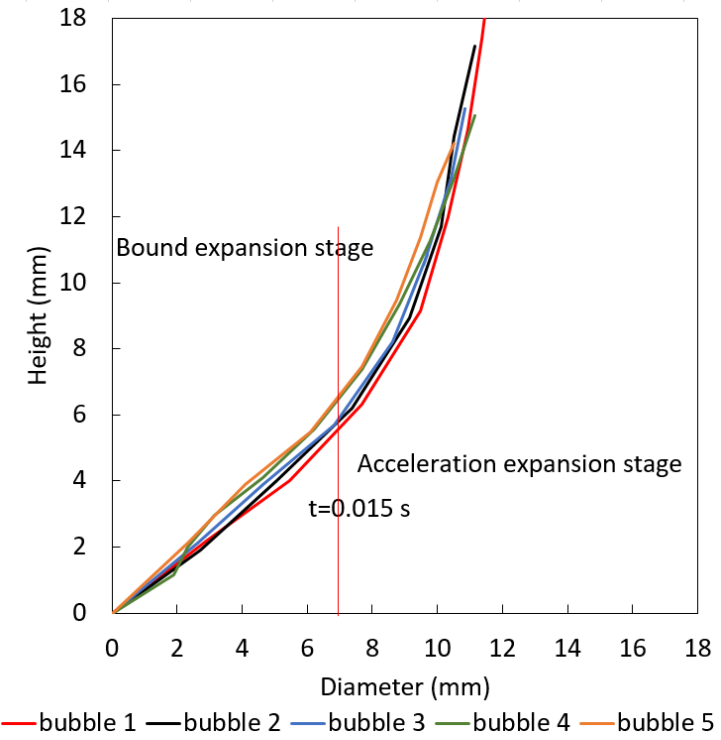

Fig. 7. Correlation between bubble diameter and height at $2 \mathrm{~m} / \mathrm{s}$

\section{B. Bubble performance after detaching}

The bubble location at inlet air speed of $1 \mathrm{~m} / \mathrm{s}$ with orifice diameter of $2 \mathrm{~mm}$ is shown in Fig. 8. The time is set to 0 when the bubble is first detached from the orifice. In total, 5 bubbles are captured to calculate the bubble's rising speed . The location signals fluctuated and were further processed to ascertaina trendline behaviour. Three distinct regions are observed as shown in Fig. 8. In region a and c, the location is shown to be 
linear to time, suggesting that the bubble is rising at a constant speed. In region $b$, the bubble first speeds up, and then slows down gradually. The linear regions are zoomed seperately as shown in Fig. 9 and Fig. 11. The averaged slopes of region $a$ and $c$ are 0.244 and 0.302 respectively. The bubble rising speed in region a is similar to the speed of the bubble centre before detaching.

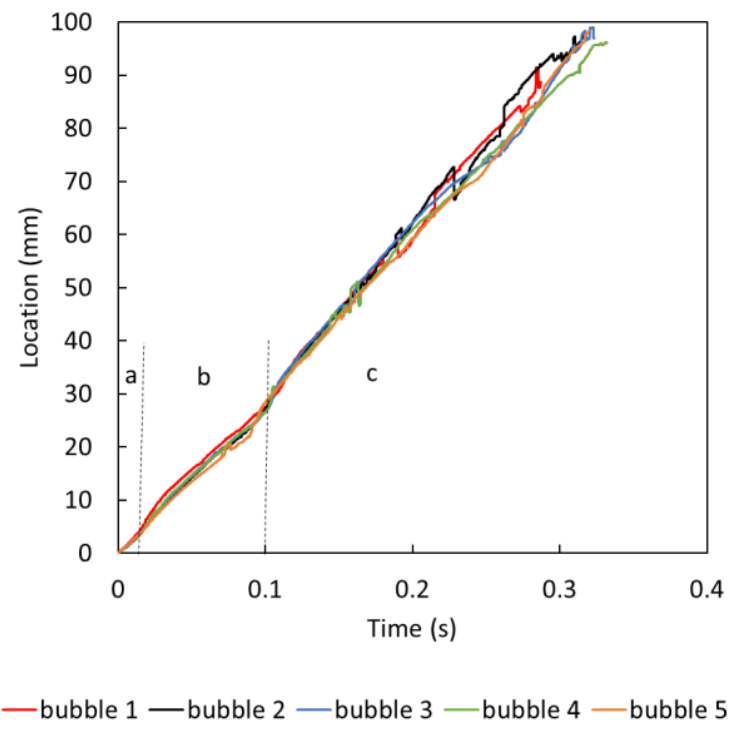

Fig. 8. Bubble location for orifice diameter of $2 \mathrm{~mm}$ at $1 \mathrm{~m} / \mathrm{s}$

In region $a$, the bubble is just detached. The upper part of the detached bubble keeps it shape and speed due to inertia, while the lower part of the bubble shrinks rapidly upwards due to immediate disappear of the drag force from the bubble neck. The development of the bubble shape is shown in Fig. 10. As maximum diameter is set as the capturing point (red dot as in Fig. 10), the captured speed $0.24 \mathrm{~m} / \mathrm{s}$ is representative of the rising speed of the upper part. That is also the reason why similar air bubble rising speed is observed at region $a$ and before detachment.

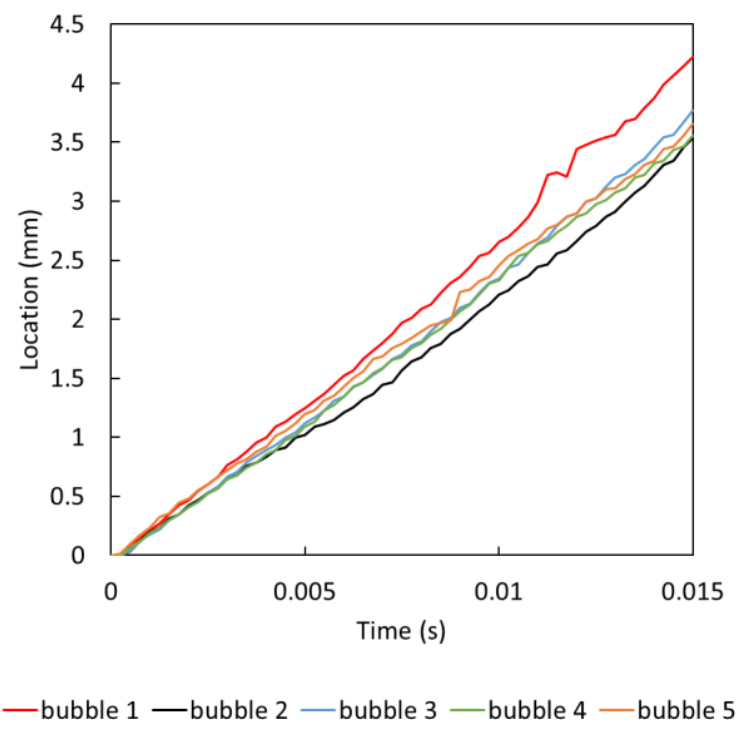

Fig. 9. Region a of orifice diameter of $2 \mathrm{~mm}$ at $1 \mathrm{~m} / \mathrm{s}$

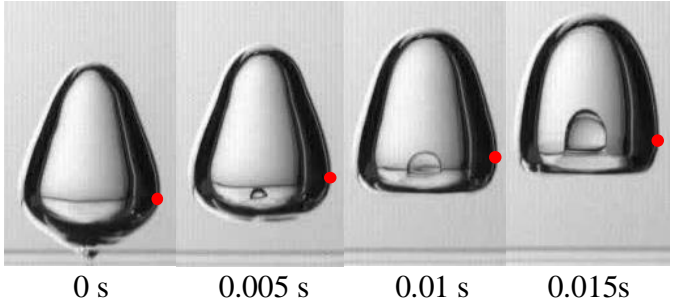

Fig. 10. Development of bubble shape in region $a$

The bubble reaches a new force balance by interactions with the surrounding liquid in region $c$. The forces acting on the bubble are buoyancy force $F_{b}$, inertia force $F_{i}$ and drag force $F_{d}$.

$F_{b}=F_{i}+F_{d}$

where $F_{b}$ is the buoyance force, $F_{i}$ is the inertia force and $F_{d}$ is the drag force. The drag force due to the wake of the previous bubble is negligible. where

$$
\begin{aligned}
& F_{b}=\left(\rho_{l}-\rho_{g}\right) g V \\
& F_{i}=\rho_{g} g V \\
& F_{d}=\frac{1}{2} \rho_{l} \frac{\pi}{4} D_{b}^{2} C_{D}\left(\frac{d s}{d t}\right)^{2}
\end{aligned}
$$

where $d s / d t$ is the rising speed of the bubble center. $C_{D}$ is the drag coefficient.

$\frac{d s}{d t}=\sqrt{\frac{8 g V\left(1-\frac{\rho_{g}}{\rho_{l}}\right)}{\pi D_{b}^{2} C_{D}}}$

When liquid density is much larger than the air density, the above equation can be rewritten as:

$$
v_{\text {bubble }}=\sqrt{\frac{8 g V}{\pi D_{b}^{2} C_{D}}}
$$

Hence the bubble rising speed is shown to be linear to the bubble diameter, which means when the bubble is rising at a constant speed, the bubble diameter does not change with time. However, the bubble size is not easy to be captured due to the constant changes of the bubble shape.

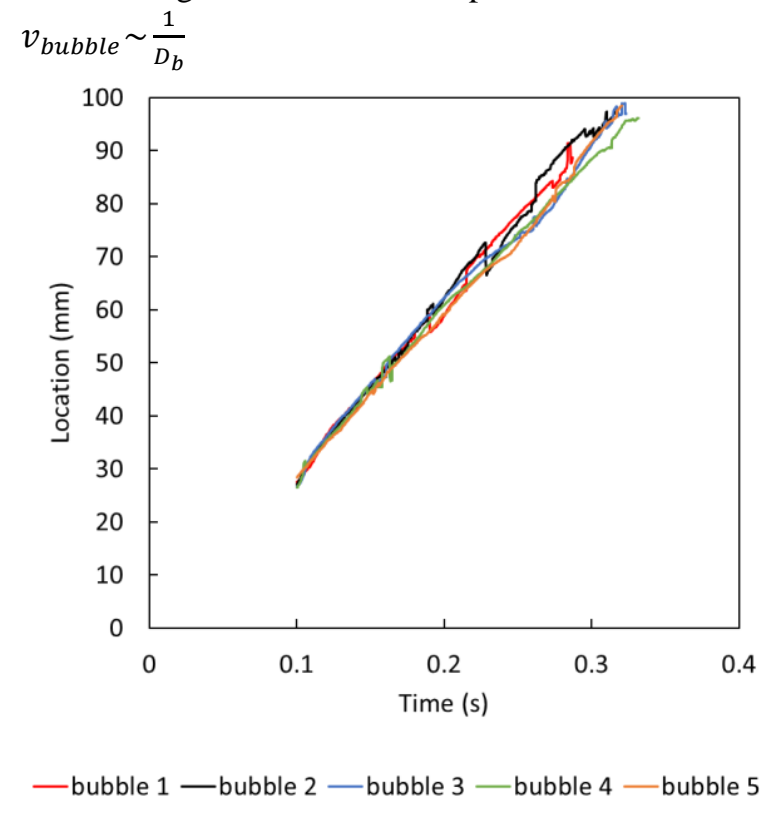

Fig. 11. Region c of orifice diameter of $2 \mathrm{~mm}$ at $1 \mathrm{~m} / \mathrm{s}$ In order to validate the above equation, the size of the bubble 
is measured for the case of orifice diameter of $2 \mathrm{~mm}$ at different air inlet velocities from $1 \mathrm{~m} / \mathrm{s}$ to $3.5 \mathrm{~m} / \mathrm{s}$ after the bubble is just detached from the orifice. Both the averaged horizontal and vertical diameters are shown in Fig. 12. It can be observed that the horizontal diameter does not change significantly with the air inlet velocity, while the vertical diameter increases with the air inlet velocity. The slope of the vertical diameter vs air inlet velocity curve reduces with the air inlet velocity. The same conclusion was also reported by other studies [4].

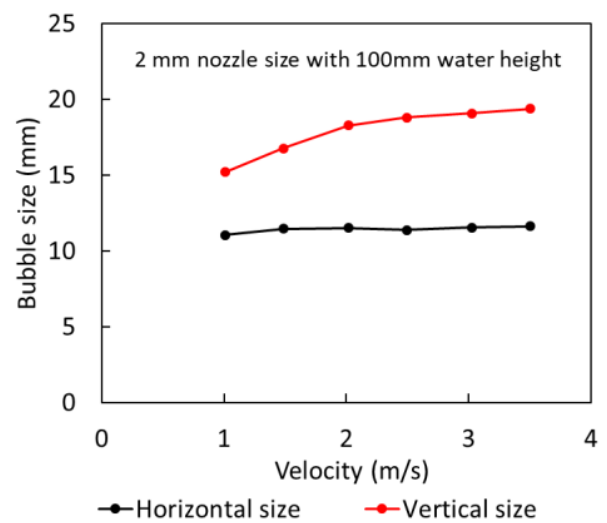

Fig. 12. Bubble size of case with orifice diameter of $2 \mathrm{~mm}$

At higher air inlet velocity, the locaton data captured is more unstable with the same orifice diameter as shown in Fig. 13 and Fig. 14. When the air inlet velocity increases to $4 \mathrm{~m} / \mathrm{s}$, air bubble collision starts to occur where some bubbles coalesce while others tend to break up into two or more small bubbles, resulting to very unstable vertical locations captured. Some of the coalescence/breakup phenomenon are shown in Fig. 15. For case $a$, the bubble breaks up from the bottom of the bubble; For case $b$, bubbles merge; for case $c$, the bubble is splited by the water sucted into the bubble; for case $d$, two bubbles merge, and then break up.

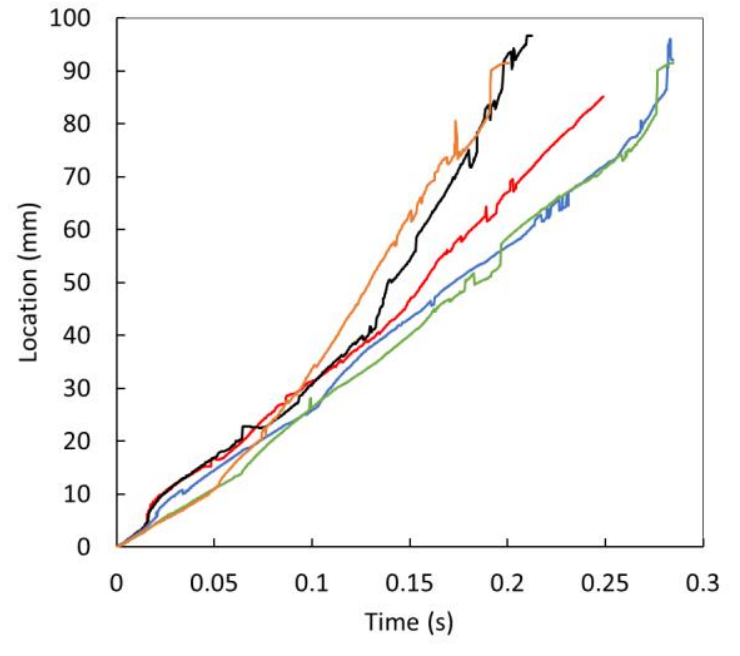

—bubble 1 - bubble 2 - bubble 3 - bubble 4 - bubble 5

Fig. 13. Bubble location for orifice diameter of $2 \mathrm{~mm}$ at $2 \mathrm{~m} / \mathrm{s}$

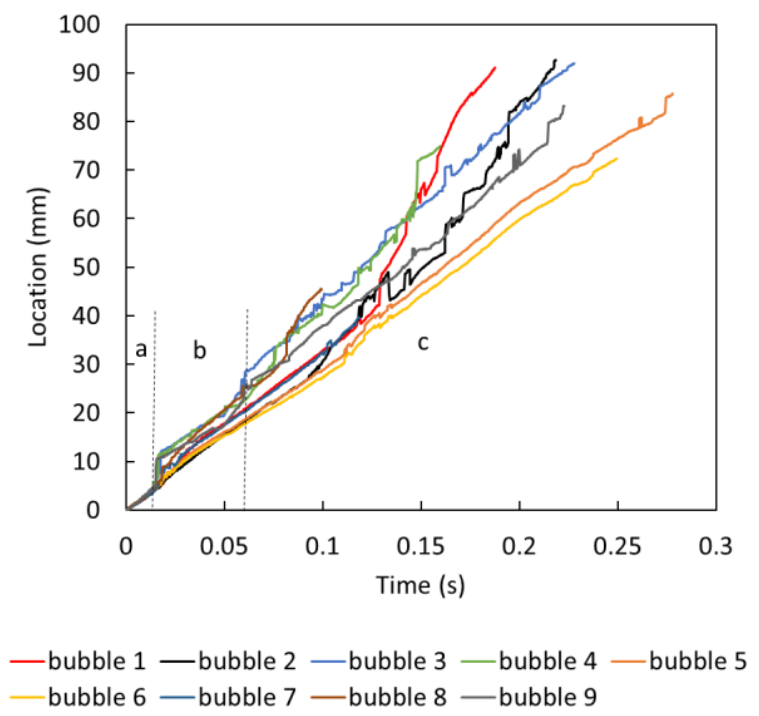

Fig. 14. Bubble location for orifice diameter of $2 \mathrm{~mm}$ at $4 \mathrm{~m} / \mathrm{s}$

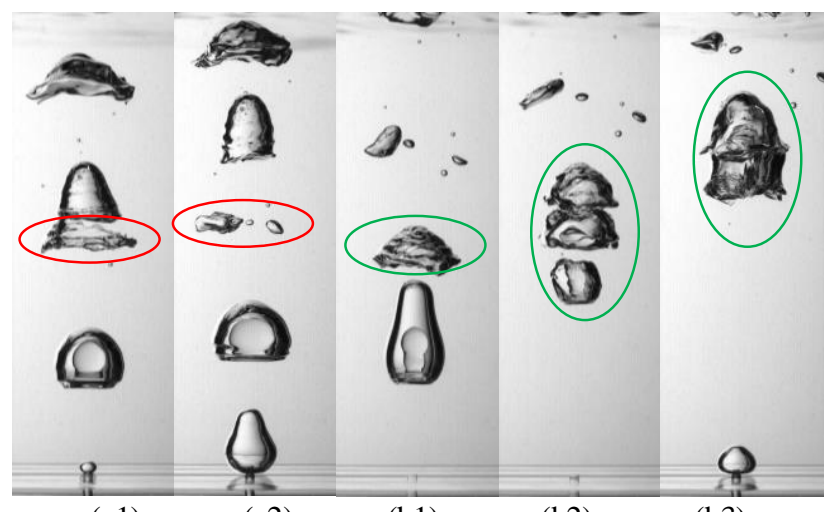

(a1)

(a2)

(b1)

(b2)

(b3)

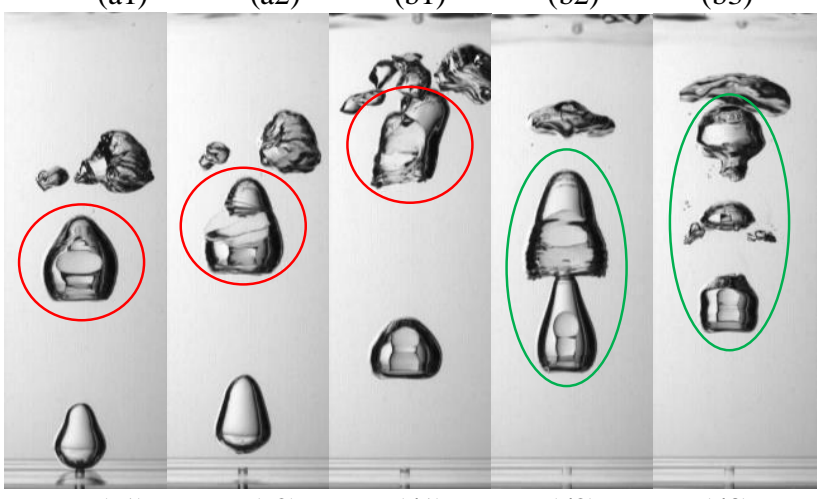

(c1)

(c2)

(d1)

(d2)

(d3)

(a) Bubble split from bottom; (b) bottom bubble split and merged with top bubble; (c) bubble separated by the water inside the bubble;

(d) two bubbles merge and split.

Fig. 15. Bubble merge/split performance at $4 \mathrm{~m} / \mathrm{s}$

For region $a$, The bubble is just detached from the orifice, and the influence of the neighboring bubble is negligible. The averaged rising speeds for $2 \mathrm{~m} / \mathrm{s}$ and $4 \mathrm{~m} / \mathrm{s}$ air inlet velocity cases are $0.23 \pm 0.03$ and $0.29 \pm 0.03 \mathrm{~m} / \mathrm{s}$. For region $b$ and $c$, the signals are unstable, and the rising speed is very hard to be consolidated, especially when mergingor spliting of the bubbles occur. 


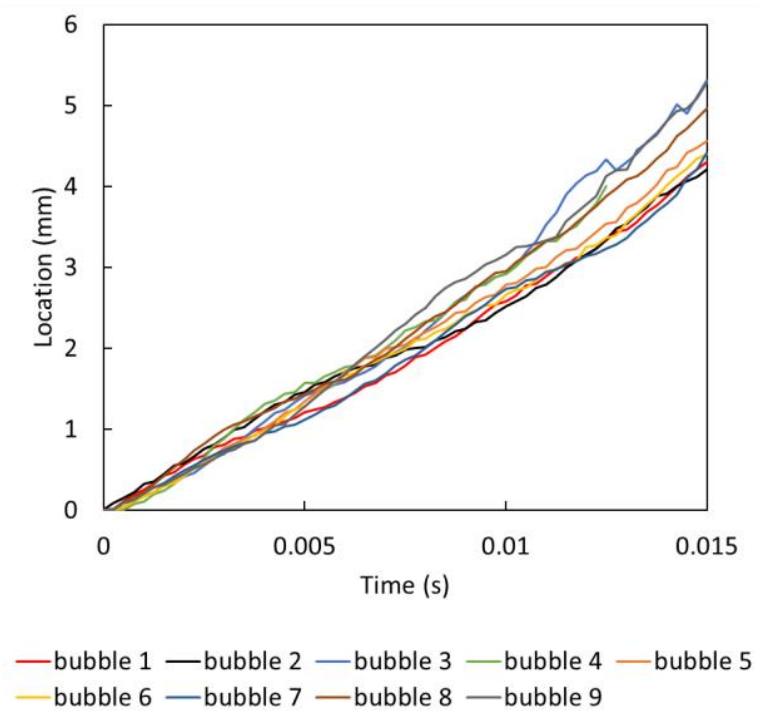

Fig. 16. Region $a$ of orifice diameter of $2 \mathrm{~mm}$ at $4 \mathrm{~m} / \mathrm{s}$

\section{V.CONCLUSIONS}

Experimental studies were performed to investigate the bubble formation characteristics before and after detachment. Before detachment, based on the correlation between horizontal diameter and vertical diameter, two stages are identified, namely bound expansion stage and acceleration expansion. In the bound expansion stage, the bubble rises at a constant speed, while expands uniformly. In the acceleration expansion stage, the bubbles still rising at a constant speed, while expands speed gradually slows down. The criteria time between the two stages at air inelt velocity of $1 \mathrm{~m} / \mathrm{s}$ is $0.2 \mathrm{~s}$, while 0.15 at air inlet velocity of $2 \mathrm{~m} / \mathrm{s}$, indicating the higher the air inlet velocity, the short is the bound expansion stage.

After detachment, the bubble rising behavior is observed to be classified into three regions. In the first and third regions, the bubble rising speed is constant, while in the second region, the bubble rising unsteadily. At detachment time, the bubble size in the horizontal direction does not change with air inlet speed, while increase slightly in the vertical direction. The averaged bubble rising speeds are $0.24 \mathrm{~m} / \mathrm{s}, 0.23 \mathrm{~m} / \mathrm{s}$ and $0.29 \mathrm{~m} / \mathrm{s}$ for cases with air inlet velocity of $1 \mathrm{~m} / \mathrm{s}, 2 \mathrm{~m} / \mathrm{s}$ and $4 \mathrm{~m} / \mathrm{s}$. This experimental results revealed the bubbles behavior at different stages and would be useful for future computational studies.

\section{REFERENCES}

[1] Z. F. Cui, S. Chang, and A. G. Fane, "The use of gas bubbling to enhance membrane processes," J. Memb. Sci., vol. 221, no. 1-2, pp. 1-35, 2003

[2] D, Bhaga and M. E. Weber, "Bubbles in viscous liquids: shapes, wakes and velocities," J. Fluid Mech., vol. 105, pp. 61-85, 1981

[3] J. Hua, J. Lou, "Numerical simulation of bubble rising in viscous liquid," Journal of Computational Physics vol.222 no. 2, pp. 769-795, 2007

[4] F. Scargiali, A. Busciglio, F. Grisafi and A. Brucato, "Bubble Formation at Variously Inclined Nozzles," Chem. Eng. Technol., vol. 37, No. 9, pp. 1507-1514, 2014

[5] R. Clift, J.R. Grace, M.E. Weber, "Bubbles, Drops and Particles," Academic Press, New York, 1978

[6] L. Zhang, M. Shoji, "Aperiodic bubble formation from a submerged orifice,” Chem. Eng. Sci., vol. 56, pp. 5371-5381, 2001
[7] A. A. Kulkarni and J. B. Joshi, "Bubble Formation and Bubble Rise Velocity in Gas-Liquid Systems: A Review," Ind. Eng. Chem. Res., vol. 44, pp. 5873-5931, 2005

[8] P. Hanafizadeh, J. Esraghi, E. Kosari and W.H. Ahmed, "The effect of Gas Properties on Bubble Formation, Growth, and Detachment," Particul. Sci. Technol., vol. 33, no. 6, pp. 645-651, 2015

[9] B. Wang, K. Zhang and R.W. Field, "Optimization of aeration variables in a commercial large scale flat sheet MBR operated with slug bubbling," J. Memb. Sci., Vol. 567, pp. 181-190, 2018 